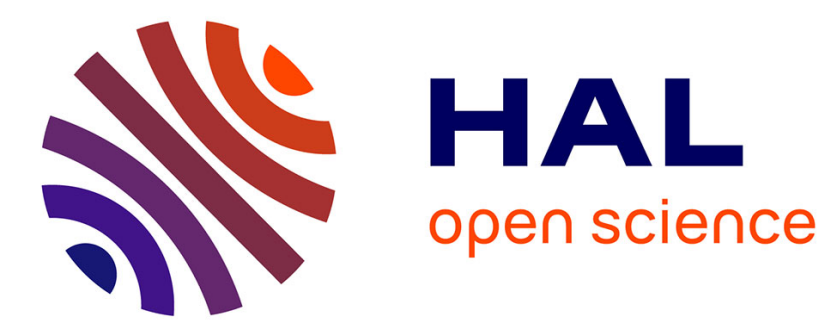

\title{
QoS-aware routing for real-time and reliable wireless sensor network based Smart Grid NAN communications
}

\author{
Mouna Rekik, Nathalie Mitton, Zied Chtourou
}

\section{To cite this version:}

Mouna Rekik, Nathalie Mitton, Zied Chtourou. QoS-aware routing for real-time and reliable wireless sensor network based Smart Grid NAN communications. SmartWorld 2017 - IEEE Smart World Congress , Aug 2017, San Francisco, United States. hal-01524382

\section{HAL Id: hal-01524382 \\ https://hal.inria.fr/hal-01524382}

Submitted on 18 May 2017

HAL is a multi-disciplinary open access archive for the deposit and dissemination of scientific research documents, whether they are published or not. The documents may come from teaching and research institutions in France or abroad, or from public or private research centers.
L'archive ouverte pluridisciplinaire HAL, est destinée au dépôt et à la diffusion de documents scientifiques de niveau recherche, publiés ou non, émanant des établissements d'enseignement et de recherche français ou étrangers, des laboratoires publics ou privés. 


\section{QoS-aware routing for real-time and reliable wireless sensor network based Smart Grid NAN communications}

\author{
Mouna REKIK \\ IFSTTAR, COSYS, LEOST, France \\ Inria, France \\ E-mail: mouna.rekik@ifsttar.fr
}

\author{
Nathalie MITTON \\ Inria, France \\ Email: nathalie.mitton@inria.fr
}

\author{
Zied CHTOUROU \\ Digital Research Center of Sfax, Tunisia \\ Email: ziedchtourou@gmail.com
}

\begin{abstract}
The deployment of Information and Communication Technologies (ICTs) within the conventional power grid has enabled new Smart Grid (SG) features and services. In this work, we focus on data routing within the distribution segment of the SG. We study communication requirements within this part of the power grid and we propose a classification of the data traffic based on Neighbourhood Area Network (NAN) communication requirements in terms of delays and reliability. Then, we introduce QoSGRACO, a routing protocol which takes account of the Quality of Service (QoS) of NAN's traffic by using coloured pheromones ant colonies. We show, through simulations, that QoS-GRACO is able to satisfy NAN's requirements, especially in terms of delay and reliability.
\end{abstract}

Keywords-Smart Grid; Neighbourhood Area Network; Geographic Routing; Ant Colony Optimization; QoS

\section{INTRODUCTION}

The future power grid, the Smart Grid (SG), implements bidirectional communications between its different domains, including generation, transmission, distribution, customers, service providers, operations and markets. Each domain interacts with the others to accomplish different SG applications. The deployment of Information and Communication Technologies (ICTs) throughout the power grid infrastructure will solve legacy problems that used to prevent implementation of smart services such as Advanced Metering Infrastructure (AMI) applications, Demand Side Management (DSM), the integration of Distributed Energy Resources and Storages (DERSs), Distribution Automation (DA) and electric transport.

The distribution segment is central to the SG. It is where the customer and the utility facilities meet. Open SG Network Task Force provided in [1] a SG conceptual actors/data flow cross domain network diagram, showing high concentration of communication traffic at this level. Data traffic generated by a single application may require different Quality of Services (QoSs) depending on the types of data exchanged and even the grid state, whether it is normal or critical. Moreover, coexistence of multiple applications using the same network, added to transmission of data of different natures and different requirements on reliability and end-to-end delay, introduces additional constraints on the routing process. Therefore, QoS data routing is recommended at Neighbourhood Area
Network (NAN) segment of the SG. Several QoS-aware routing protocols have been proposed for SG NAN in the literature. Some of these studies propose new QoS routing protocols such as GATAS [2], an on-demand routing protocol that uses a genetic algorithm combined with a flooding-like routing mechanism. Others are modified versions of existing routing protocols, such as HWMP, the path selection protocol of IEE802.11s [3]-[8] and the RPL standard [9]. Although most of these studies provide solid and valuable solutions for QoS-aware routing in the SG NAN, none of them takes account of the particularities of such networks. Actually, NAN devices are deployed in outdoor harsh environmental conditions, with dynamic topology changes, instability of wireless links, interference and fading [10] [11]. Thus, proactive and tree-based-routing protocols such as HWMP and RPL, as well as flooding-like routing protocols, are not suitable for NANs since they generate high overhead in terms of control packets, which can heavily impact the performances of the network.

Geographic routing is a good alternative to be used over NAN. This is justified by its memory-less, distributed and localized features which improve the flexibility and the scalability of the network. In this context, GPSR [12] is considered as a strong candidate for NAN. In fact, in addition to the advantages of geographic routing, GPSR guarantees packet delivery. However, several studies demonstrated that GPSR may generate significant overhead in the cases of multipoint-to-point (MP2P) and pointto-multipoint (P2MP) communications [13]. In a previous work [14], we proposed GRACO, a geographic routing protocol based on ant colonies that offers scalability, selfhealing and self-organization capabilities and supports MP2P, P2MP and point-to-point (P2P) communication modes with low complexity and overhead added compared to GPSR [15]. All these features make GRACO suitable for NAN. Nonetheless, it does not support routing data with different QoS requirements. Based on these observations, the contribution of this paper is two-fold: - We propose a classification of NAN traffic based on reliability and end-to-end delay requirements. This classification will be used to differentiate data traffic for the routing process.

- We introduce a new geographic routing protocol, QoSGRACO, inspired from GRACO and taking account of 
NAN traffic QoSs. We use coloured pheromones ant colonies to support multiple QoSs geographic routing. QoS-GRACO searches for paths with different qualities in terms of end-to-end delay and reliability, and adjusts the routing process depending of the QoS required by the data. Simulations show that QoS-GRACO is able to satisfy NAN's communication requirements and allows for different classes of QoS.

The rest of the paper is organised as follows. Section II provides a description of NAN data traffic, and discusses their QoS requirements. Section III introduces the notations and the system models used in the paper. Section IV briefly details GRACO routing protocol. The functioning of the proposed QoS-GRACO routing algorithm is presented in Section V. In Section VI, we evaluate the performances of QoS-GRACO through simulations. Finally, in Section VII, conclusions are outlined.

\section{QoS REQUIREMENTS OF NAN TRAFFIC}

In this section, we analyse the expected traffic in the NAN in order to highlight its QoS requirements.

\section{A. NAN traffic description}

In order to support new SG functionalities, four types of data packets are used to carry data over the NAN: command, data, alert and alarm [1]. Command packets are usually queries to request some data or measurements or control commands for network devices. The class of data packets covers periodic measurements, responses to commands and packet acknowledgement (ack). The alert packets are generally notifications, such as a device status change notifications or that it operates outside its normal limits, such as the system voltage drops under a predetermined level. Alarm packets carry more critical data than alert packets, they notify a malfunction or improper operation of certain devices when threshold conditions are exceeded due to failures such as power outages and blackouts.

NAN traffic depends on the system status. Normal traffic mainly includes a fixed scheduling traffic as periodic measurements and meter reading, in addition to fault detection and asynchronous events control traffic, in MP2P communications, data and command traffic in P2MP communications, and diverse P2P traffic. Some normal functioning traffic may be stopped during critical conditions until operations are back to normal conditions, such as firmware updates and configuration commands. However, other normal conditions traffic must be carried during critical conditions, such as periodic measurement used for some services related to critical operation. On the other hand, during critical conditions, some extra load may be added, such as additional traffic generated by Smart Meters (SMs) for the re-registration with AMI head end or Meter Data Management System (MDMS) after a blackout or failure.

\section{B. Traffic priority levels}

Based on the traffic analysis presented in the previous section, we propose five priority levels for NAN traffic, described here from the highest to the lowest:

1) High Priority Critical traffic : mainly for infrastructure protection. The data exchanged is related to power outages and critical failures alarms, including some from DA services (for example when a device fails to perform certain task or exceeds a predefined threshold) and security alarms, potentially producing dangerous malfunctions in the distribution network, especially in the case of cascading several anomalies.

2) Critical conditions traffic: data exchanged during network operation under fault conditions, such as fault isolation and network restoration traffic, measurements and data required for certain treatments needed during critical conditions.

3) High priority traffic of normal operation: alerts and notifications on change of status of certain devices, or operating outside normal limits.

4) Normal traffic operation: as periodic measurement packets exchanged to perform normal operations as a request to measure a service switch control.

5) Background traffic: firmware updates and configuration of network devices.

Based on this classification, we designed QoS-GRACO, a routing protocol suitable for SG NAN that accounts for its QoS requirements.

\section{Delay requirements}

Respecting delay requirements is a very critical issue for NAN applications. Indeed, some operations require real time measurements to control the grid component, especially in case of failure, malfunctioning or any other abnormal behaviour detection. As a result, the network performances depend on whether the data is transmitted within the required delays. The end-to-end delay requirements depend on the nature of the data to deliver; data that directly affects system operations, such as monitoring and control data, must have extremely low delay of the order of tens of milliseconds (20ms [16], [17]), while other data, such as firmware updates, require longer delays, up to hours or even days [1]. Several studies were performed to determine the delay tolerated by each application. Some studies have specified it in minimum allowance [16], [17], others have opted to set the maximum allowance [1].

For AMI applications, required delays vary from $250 \mathrm{~ms}$ for critical data to $1 \mathrm{~s}$ for periodic measurements [16]. The experts of U.S. Department of Energy (DOE) suggest that the delays of AMI data is between $2 \mathrm{~s}$ and $15 \mathrm{~s}$ [18]. Others suggest that these delays can be tolerated up to few minutes or hours [1].

For DSM applications, communication requirements of the services may vary depending on the sophistication desired by service providers; simply more tolerable with requirements such as direct load control by simply sending a shut-down command to a device (an air conditioner or heating of hot water), the most advanced to the most critical applications requirements, such as services that allow equipment to respond to dynamic conditions on the grid, consumption shifting in real time in response to dynamic pricing signals, the availability of electricity supplied by the distributed generation resources in the neighbourhood. Delay values required by DSM applications range from $500 \mathrm{~ms}$, as a minimum allowance, to $15 \mathrm{~s}$ as a maximum allowance [1], [16]. 
Renewable electricity is variable by nature and likely to be even more unpredictable when used on a small scale. Thus, exchanging measurements and control signals in near real-time is needed to ensure a seamless integration into the network. Indeed, by providing instantaneous information on the electricity production on distribution network facilities, the utility will be able to efficiently allocate this energy. Estimates of the delays required for DERS applications range from $300 \mathrm{~ms}$ to $2 \mathrm{~s}$, while in critical conditions the delay decreases around tens of milliseconds. For Electric Vehicle (EV) application, delay estimations vary between $2 \mathrm{~s}$ and $5 \mathrm{~min}$ [18].

DA applications allow some of the most critical network services: fault detection, isolation and restoration. Reception of notifications and alerts about abnormal behaviour or measurements in sufficient delays enables utilities to anticipate and prevent, as much as possible potential failures. In the worst case, where a failure occurs, fast and precise detection and communication will reduce the impact, the frequency and duration of outages. The utility is then able to move quickly to isolate the fault, limit its impact, repair and trigger automatic restoration to provide power through another circuit if necessary. Other DA non-critical services can tolerate longer delays than the critical events, such as simple data measurements used to improve control of capacitors and voltage regulators, which helps keep the circuit voltage and reduce energy waste. In short, the time required by DA applications should not exceed $1 \mathrm{~s}$ according to U.S.DOE [18]. In [16], the minimum delay allowances are as follows: $100 \mathrm{~ms}$ for critical events, $200 \mathrm{~ms}$ for critical commands, $500 \mathrm{~ms}$ for non-critical operations data. Experts from Open SG Task Force estimate the maximum delay allowance to $3 \mathrm{~s}[1]$.

Generally, traffic with lower time requirements is of higher priority level, and vice versa.

\section{Reliability requirements}

The network reliability is defined as the probability of the availability of a network connection between both end parts. It depends on many factors, including the communication medium, the environment (such as underground or outdoor), weather and physical conditions of the network components. It is also strongly influenced by the frequency of nodes and links failures in the network, as well as the repair time. Due to the nature of the data exchanged over the communication networks of the SG, the reliability requirements are very high. Indeed, the experts of the U.S.DOE have estimated the required reliability values between $99 \%$ and $99.999 \%$ for NAN applications [18]. A reliability of $99.999 \%$ for a network connection results in an outage probability of 0.00001 network (due to failures in the network) [16]. Often this unavailability is defined as the period of time where the network will not be available over a year. Thus, for a reliability of $99.999 \%$, the unavailability $(0.00001 \times 365 \times 24 \times 60)$ is less than 5.3 minutes on average per year [16]. The network reliability can be improved by using multiple physical paths.

The reliability of data transmission is the probability that the data is delivered without failure over a period of time. Open SG Network Task Force experts estimated the reliability requirements of normal operation data greater than $98 \%$ (greater than $90 \%$ for some services such as meter reading) and greater than $99 \%$ for error notifications, alerts, alarms and data in response to specific commands or treatments [1].

To conclude, at the routing level, the reliability of data transmission over the NAN may be improved by using three concepts: 1) multi-path routing with multiple disjoint paths between two end points, 2) data routing using fast and efficient recovery mechanisms in the case of failure and 3) reliability aware routing using a routing mechanism that takes account of the reliability of connections when forwarding packets.

\section{Notations AND System MODELS}

We model the NAN as a wireless network composed of nodes such as SMs, field devices and sensors. We assume that all nodes are aware of their location. This can be configured during the deployment within the power grid. The network is modelled as a directed graph $G=(V, E)$ composed of a finite set of nodes $V$ and links $E$. We note $|u v|$, the Euclidean distance between two nodes $u$ and $v$ is noted. A wireless link between nodes $u$ and $v$ exists if $u$ and $v$ are within transmission range of each other, i.e. $|u v| \leqslant R$, where $R$ represents the transmission range. The physical set of nodes in transmission range of node $u$ is noted $N(u)$ and called the neighbourhood of node $u$, i.e. $N(u)=\{v \in V$ such as $|u v| \leq R\}$. We note $|N(u)|$ the number of neighbours of $u$. We also define $N_{D}(u)$ the subset of nodes in $N(u)$ closer to node $D$ than $u$ itself, i.e. $N_{D}(u)=\{v \in N(u)$ such as $|v D| \leq|u D|\}$. The directed link from a node $u$ to a node $v$ is noted $\overrightarrow{u v}$.

In the following, we call "current node" the node trying to route a packet and we use NextNode to refer to the next hop on the path of a packet. We note $C(A, r)$ the circle $\mathrm{C}$ of radius $\mathrm{r}$ and centred at $\mathrm{A}$. For two nodes $\mathrm{A}$ and $\mathrm{B}$, the circle $C(A,|A B|)$ is of radius $|A B|$ and centred at $\mathrm{A}$. We call progress zone of a node $u$ toward a destination $D$, the intersection of circles $C_{1}(u, R)$ and $C_{2}(D,|D u|)$. Nodes from this zone are called progress neighbours, the set of these nodes is denoted $N_{D}(u)$.

\section{BACKGROUND : GRACO}

QoS-GRACO, the contribution of this paper is a modified version of the previous GRACO protocol. This section provides a short overview of GRACO in order to set the differences between both protocols. GRACO [15] is a geographic routing algorithm that combines a pheromoneassisted greedy forwarding mode and an ant colony optimization (ACO) based recovery mode. A data packet is first routed using the plain greedy forwarding [19], forwarding the packet to the closest neighbour to the destination. When the packet reaches a node $K$ with no progress neighbour to the required destination, it is stuck since $K$ cannot find a node closer to the destination than itself. As such, $K$, called the stuck node, launches a recovery mode using exploratory ant-like packets called Fants in order to search for an alternative path to a node that is closer than the stuck node to the destination, or to the destination itself. When a Fant finds a path, a 
backward ant (Bant) is sent back to $K$ to mark the path found by dropping pheromone trails. The stuck node waits for a Bant to arrive. If so, a path is established and data packets can be sent. The data packet will be routed using the dropped pheromone trails until reaching an unstuck node, and then switch to greedy forwarding again until its destination or another stuck node, in such a case, the same mechanism is applied again. The pheromone trails for a destination $D$ will be re-used later to route next data packets: when $U$ has a data packet for $D$, it first searches for pheromone trails for $D$, if such pheromone exists, $U$ uses these pheromones to decide the next node to which forward the packet, otherwise it tries the plain greedy forwarding mechanism.

In the next section, we propose a modified version of GRACO that takes account of the data QoS requirements based on the classification proposed in Section II.

\section{QOS-AWARE GEOGRAPHIC GREEDY FORWARDING WITH} ACO BASED RECOVERY

In order to adapt the routing process to the specific case of NANs, we propose a new QoS-aware routing protocol that takes QoS requirements into account. It consists in routing data depending on the QoS class of traffic to which they belong. In addition to this, a priority treatment is also carried out if necessary. If two packets of the same class arrive at a node, the one with the highest priority is sent first. The priority level depends on the nature of data as described in Section II-B.

QoS-GRACO is QoS a multi-path geographic routing protocol that relies on two modes: a modified greedy mode and a recovery one. Like in GRACO, the first mode is a pheromone assisted greedy forwarding. However, it is no longer the plain greedy but one that takes account of the required QoS. The recovery mode uses coloured pheromones, each colour corresponds to a class of traffic. The overall operation of QoS-GRACO is summarized in Algorithm 1. Each node receiving a packet first attempts to progress it using pheromone trails, if a convenient pheromone exists. If so, that means that a recovery has already been launched to look for a route to the same destination with an appropriated QoS. Otherwise, the node tries to forward the packet using the plain greedy mode, if this is not possible because of a physical void it triggers the recovery mode. Both modes are detailed below.

\section{A. QoS Model}

QoS is generally defined as a set of service requirements that must be supported. QoS metrics can be classified as additive, concave and multiplicative metrics. Let $m(u, v)$ a QoS metric characterizing $(u, v)$, a path connecting node $u$ to node $v .\left(u, u_{1}, u_{2}, \ldots, u_{k}, v\right)$ is the set of nodes belonging to the path $(u, v)$. The metric $m$ is: - additive if $m(u, v)=m\left(u, u_{1}\right)+m\left(u_{1}, u_{2}\right)+\ldots+m\left(u_{k}, v\right)$. The end-to-end delay is an additive metric;

- concave if $m(u, v)=\min \left\{m\left(u, u_{1}\right), m\left(u_{1}, u_{2}\right), \ldots, m\left(u_{k}, v\right)\right\}$. The reliability and the bandwidth are concave metric;

- multiplicative if $m(u, v)=m\left(u, u_{1}\right) \times m\left(u_{1}, u_{2}\right) \times \ldots \times$ $m\left(u_{k}, v\right)$. The probability of loss is a multiplicative metric.

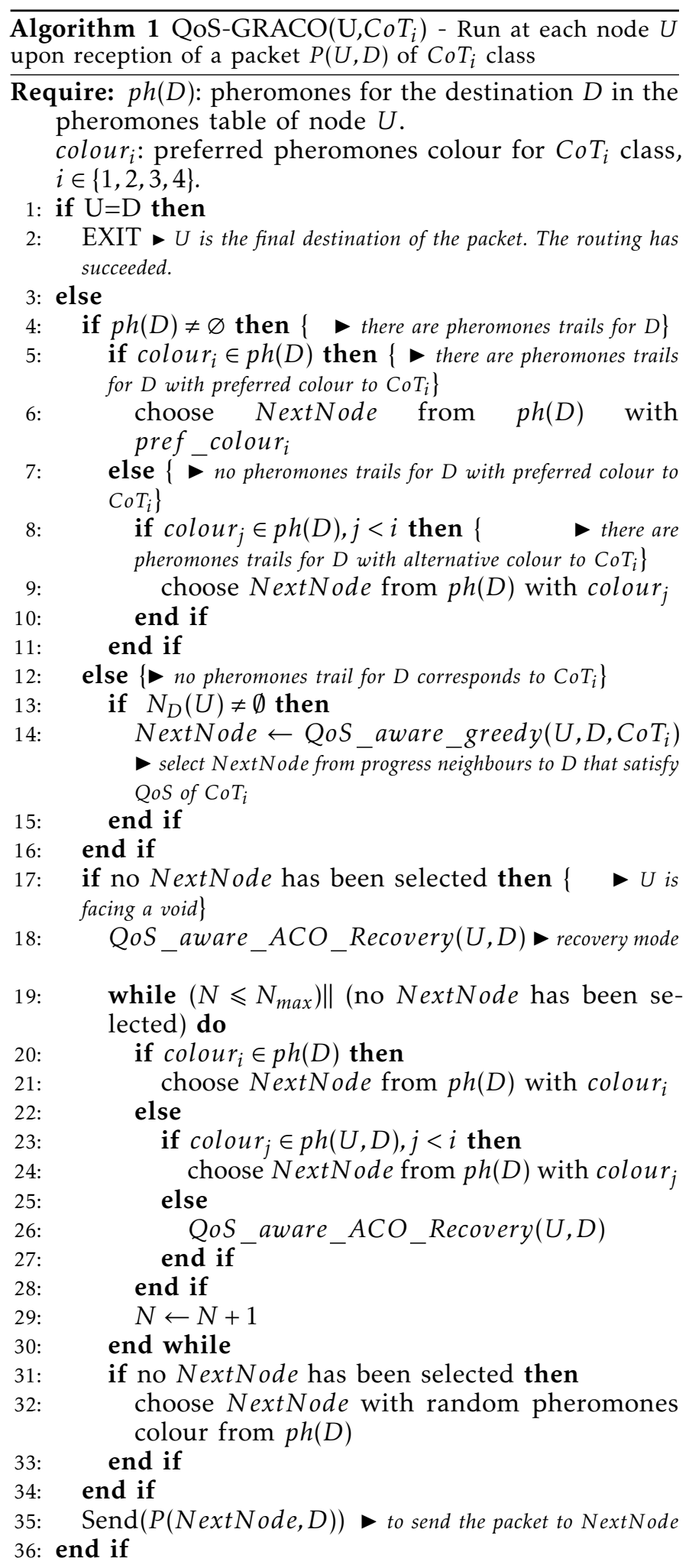

In this work, we consider end-to-end delay and reliability. Using only local information, each node is able to calculate the quality of those links as follows:

- the end-to-end delay of a link $\overrightarrow{A B}$ : is the time interval between the time the node $A$ sends the packet and the time the node $B$ receives it, including the communication 


\begin{tabular}{|l|l|l|l|}
\hline Class & Description & Delay & Reliability \\
\hline $\mathrm{CoT}_{1}$ & High priority traffic & $D<30 \mathrm{~ms}$ & $R>99 \%$ \\
\hline $\mathrm{CoT}_{2}$ & Critical operations traffic & $D<50 \mathrm{~ms}$ & $R>98 \%$ \\
\hline $\mathrm{CoT}_{3}$ & $\begin{array}{l}\text { Normal operations priority } \\
\text { traffic }\end{array}$ & $\begin{array}{l}\mathrm{D} \\
200 \mathrm{~ms}\end{array}$ & $R>95 \%$ \\
\hline $\mathrm{CoT}_{4}$ & $\begin{array}{l}\text { Normal operations and } \\
\text { background traffic }\end{array}$ & $\mathrm{D}<1 \mathrm{~s}$ & $R>90 \%$ \\
\hline
\end{tabular}

TABLE I: Classes of traffic

channel delay, the radio propagation delay, the standby delay and the protocol processing time,

- the reliability of a link $\overrightarrow{A B}$ : is the rate of successfully transmitted packets on the link.

Based on NAN traffic analysis performed in Section II, we propose to classify the data traffic of the NAN into four categories called classes of traffic. Each class is characterized by QoS requirements, in terms of end-toend delay and reliability and as shown in Table I. We chose to use the estimations of minimum allowance in our classification to maximize the margin of safety. In case a packet slightly exceeds the estimated minimum delay, it remains below the maximum allowed delay.

B. Greedy Mode: QoS aware ph-assisted greedy forwarding

The greedy mode of QoS-GRACO routing protocol is mainly based on GRACO's one, ie ph-assisted greedy forwarding. However, we propose a modification of next node selection criteria to take the QoS required into account. Next node selection process of QoS-aware phassisted greedy routing consists of choosing one node from the neighbours in the positive progress area, which locally satisfies the QoS of the packet Class of Traffic (CoT). The functioning of QoS-aware greedy routing is illustrated through Figures 1, 2 and 3. Different colours are use to mark the paths (and nodes belonging to these paths) that meet the requirements each traffic class. The colour red is used to mark $\operatorname{CoT}_{1}$, the blue $\mathrm{CoT}_{2}$, the green $\mathrm{CoT}_{3}$ and finally the black $\mathrm{CoT}_{4}$.

Fig. 1 presents a routing scenario of a packet of $\mathrm{CoT}_{3}$ using QoS-GRACO greedy mode. The current node progress neighbours are coloured with the colour of the CoT for which they meet its requirements. $N_{1}, N_{2}$ and $N_{5}$ (marked in green) meet the requirements of $\mathrm{CoT}_{3}$ class, while $N_{3}$ and $N_{4}$ (marked in black in correspondence to $\mathrm{CoT}_{4}$ ) provide less restricted QoS. In this first scenario, a packet of $\mathrm{CoT}_{3}$ arrives at current node $S$. S first looks for pheromone trails (preferred or alternative) satisfying the requirements of the packet class. If no such pheromones exist, $S$ tries to progress the packet using QoS-aware greedy forwarding, that means that $S$ selects the node that meets the requirements of the class from its neighbours in the positive progress zone to $\mathrm{D}$, if there are several, as it is the case in this example presented in Fig. $1\left(N_{1}, N_{2}\right.$ and $N_{5}$ are candidates), $S$ selects $N_{2}$, which provides the maximum progress towards $\mathrm{D}$ among these nodes. The packet will be forwarded to $N_{2}$. The same treatment is initiated at each node receiving the packet until reaching its destination.

In the second scenario, presented in Fig. 2, a packet of $\mathrm{CoT}_{2}$ class arrives to $S$. $S$ starts the routing process, it finds no pheromone trails or progress neighbours satisfying the requirements of the packet. The packet is considered in a void. In this case, it is not a physical void, it is a $Q o S$ void. Consequently, $S$ triggers a recovery mode. During this mode, several routes of different qualities (hence of different colours) have been discovered. Among these routes, $S$ chooses to use the favourite colour to the packet class; i.e. the route marked in blue. In case the preferred colour does not exist, the node selects then a route with alternative colour to the CoT. We call an alternative colour to a CoT, when it meets the requirements of another more restricted class of traffic. This route can then satisfy less restricted requirements, however, the packets belonging to the corresponding class have the priority to use paths with this colour.

In the third example, shown in Fig. 3, a packet of $C_{0} T_{1}$ class arrives to $S$. When starting the routing process, $S$ finds several pheromone trails, that means that the greedy mode has previously failed, a recovery mode has been launched and pheromone trails have been already dropped to mark the paths found. For this reason, the current node uses the existing pheromone, it chooses the path marked with the favourite colour among the existing traces if it exists (as in this case the path marked in red). If it not the case, it searches for an alternative colour.

\section{Recovery mode: QoS-aware ACO based recovery}

Like in geographic GReedy routing with ACO based recovery (GRACO), QoS-GRACO recovery process takes over whenever the first phase is no more possible. This happens in cases of physical voids but also QoS voids, i.e. when the progress zone of the current node is not empty, but no neighbour of this area meets the required QoS.

GRACO-QoS uses the same concept of route establishment, the management process of cascading recoveries and the loop management process of GRACO protocol [15], using the concept of zones, Fants and Bants. However, in order to consider the QoS requirements of the classes of traffic described in Section V-A, we introduce the concept of colour to the pheromones, such as the pheromones deposited by ants become coloured based on the qualities of the paths found. The Fants calculate the quality of the path during the exploring phase. Depending on the quality of the path announced by the corresponding Fant, a Bant decides the colour and the intensity of the pheromones depending of the path suitability for a class of traffic. In its way back to the node that triggered the recovery mode, Bants mark the paths found by depositing trails of coloured pheromones at each node along the way.

Each class of traffic is associated to a specific colour, for example, a path with a delay $\leqslant 30$ milliseconds and a reliability $\geqslant 99 \%$ is more suitable for the traffic of $C_{0} T_{1}$ and it will be marked by the type of pheromone (or colour) red. Fig. 4 presents the classes of traffic $\left(\mathrm{CoT}_{1}\right.$, $\mathrm{CoT}_{2}, \mathrm{CoT}_{3}$ and $\mathrm{CoT}_{4}$ ) and the corresponding colours (red, blue, green and black).

When the node that triggered the recovery process receives a Bant, it attempts to move the data packet blocked using the path found if the pheromone colour matches the packet class of traffic. The pheromone trace 


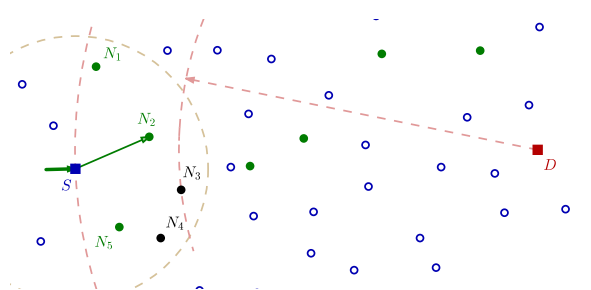

(a) $S$ forwards the packet to $\mathrm{N}_{2}$ using QoS-aware greedy forwarding.

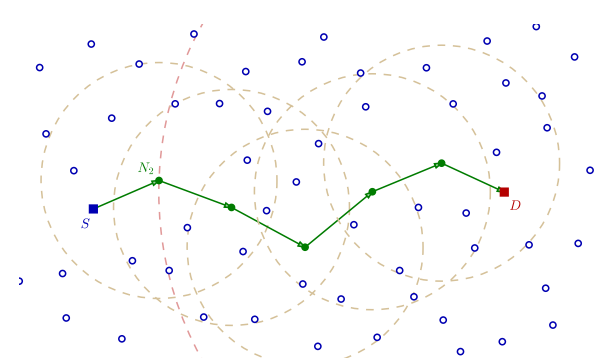

(b) The packet arrives to its destination using QoS-aware greedy forwarding.

Fig. 1: Scenario 1: routing of data packet of $\mathrm{CoT}_{3}$ using QoS-GRACO.

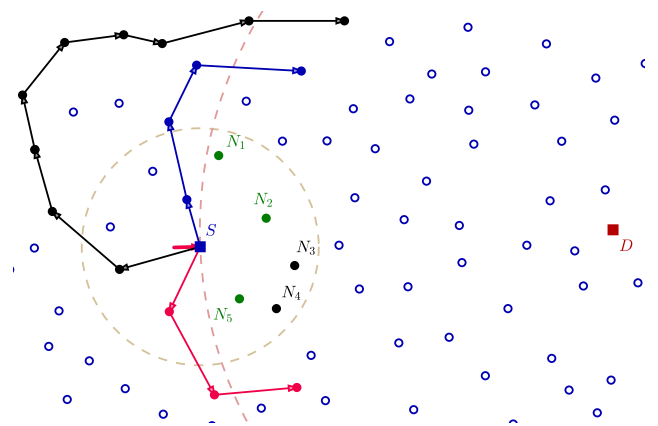

(a) $S$ tried to forward the packet using QoS-aware greedy forwarding. No progress neighbour satisfies the QoS of $\mathrm{CoT}_{2}$. $S$ launches the recovery mode.

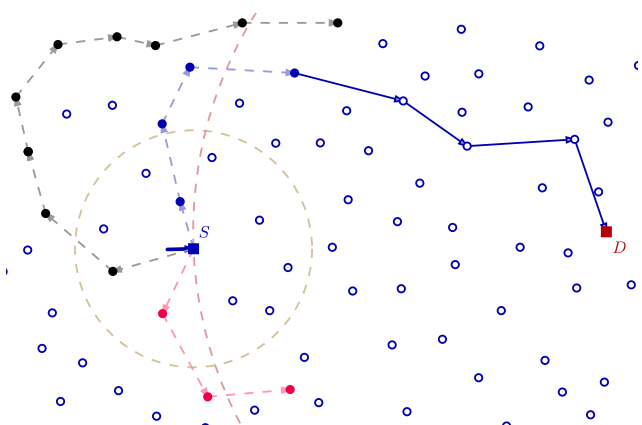

(b) The packet is forwarded using pheromone trails, then QoS-aware greedy forwarding until it arrives to its destination.

Fig. 2: Scenario 2: routing of data packet of $\mathrm{CoT}_{2}$ using QoS-GRACO.

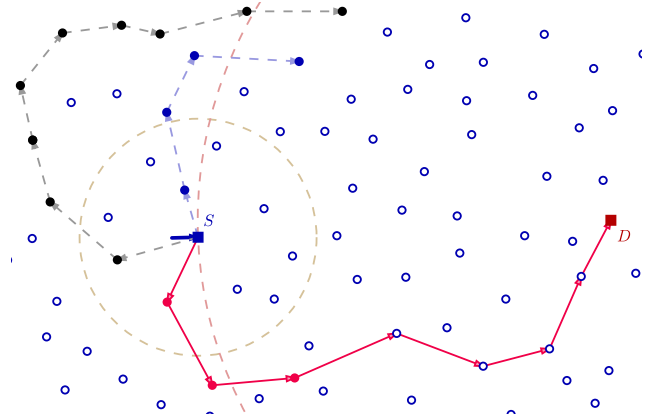

Fig. 3: Scenario 3: routing of data packet of $C_{0} T_{1}$ using QoS-GRACO. The packet is forwarded using existing pheromone trails then QoS-aware greedy forwarding until it arrives to its destination.

corresponds to a class of traffic if it is marked with preferred or alternative colour for this class, as shown in Fig. 4. Otherwise, if the trace does not match the blocked packet class of traffic, the node launches the recovery mode again. In order not to flood the network with Fant and Bant packets, which can lead to additional delay and affect the reliability of the links, we limit the number of relaunching the recovery process by a maximum of $N_{\max }$ attempts. If no running recovery process results in a suitable path, the node attempts, as well, to transmit the packet blocked using available pheromones, the selection process of the trace in this case supposes that pheromones are not coloured, so uses the route selection of GRACO.

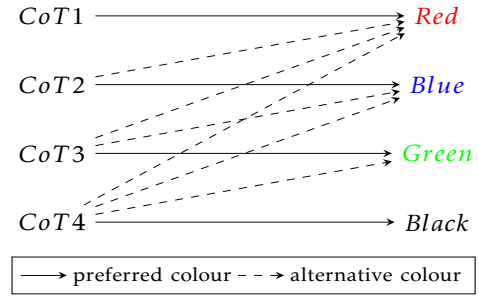

Fig. 4: Mapping traffic classes to the colours of preferred and alternative pheromones

\section{Performance evaluation}

In this section, we evaluate the performance of QoSGRACO algorithm and its relevance to the QoS requirements of SG NAN. We simulated the functioning of QoS-GRACO on connected networks with random topologies, with a number of nodes ranging from 250 to 900 in a field of $300 \mathrm{~m} \times 300 \mathrm{~m}$. The nodes have a communication range of $25 \mathrm{~m}$. The simulated networks contain necessarily communication voids of random sizes, shapes and locations. We select a set of random sources and destinations in each network in such a way that they are in different sides of a void to trigger recovery process at least once. To simulate data traffic, ten data packets of each traffic class are sent between each pair (source, destination). We measure the performances of QoS-GRACO in terms of data delivery rate, cost of sending data and end-to-end delay. The delivery rate is the ratio of the number of successfully received data packets 
to the number of data packets sent. The cost is measured as the number of packets used to deliver the data to its destination, including control packets (HELLO packets used to exchange information between the neighbours, Fants and Bants used to establish a path) and data packets. Finally, the end-to-end delay is recorded as the time interval between the time when the source has sent the first data packet and the time when the destination has received the last. The simulation parameters are summarized in Table II.

\begin{tabular}{ll} 
Parameter & Value \\
\hline Duration (s) & 3600 \\
Routing & QoS-GRACO \\
MAC & IEEE 802.15.4 CSMA-CA \\
Radio & radio_half $1 d k$ \\
Modulation & bpsk \\
Field size & $300 \mathrm{~m} \times 300 \mathrm{~m}$ \\
Communication range & $25 \mathrm{~m}$ \\
Number of nodes & $250,350,450,550,650$, \\
& $750,850,900$ \\
\hline
\end{tabular}

TABLE II: Simulation parameters

\section{A. Cost}

Fig. 5a illustrates the data delivery cost varying with the number of data packets sent. Simulation results, presented on Fig. 5a, show that the algorithm uses more control packets when delivering data belonging to traffic classes with more constrained requirements. Indeed, using realistic MAC layer, the quality of links is very variable, the first phase of the algorithm, which is the greedy phase, does not always yield to the same void, the recovery phase is, then, launched several times and in different locations during the research process. However, as shown in Fig. 5b, as the number of packets sent between the same source and the same destination increases, the cost decreases, as with more pheromone trails are deposited by previous recoveries, the routing will be performed using the pheromone and the probability to launch other recovery decreases.

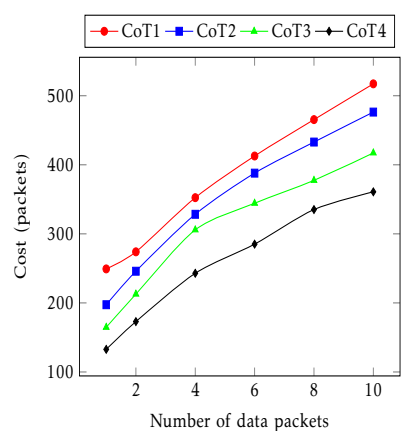

(a) Cost varying with the number of data packets sent

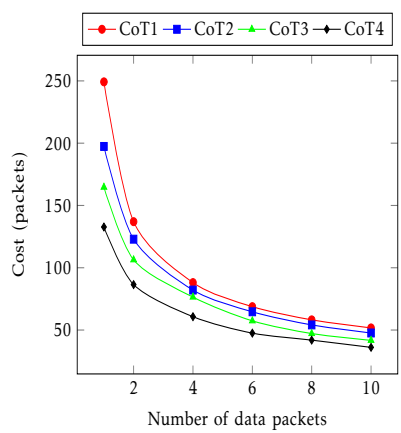

(b) Cost per packet varying with the number of data packets sent
Fig. 5: Cost - 802.15.4 MAC

Fig. $5 \mathrm{~b}$ plots the cost per packet of each class of traffic. The simulations show that delivering first data packets is always more expensive in terms of control packets than delivering the next packets sent between the same source and the same destinations. Indeed, after a while, several pheromone trails are deposited to mark the paths found during previous recoveries, and these trails are subsequently used for routing next data packets.

\section{B. End-to-end delay}

Fig. 6a present the end-to-end delay varying with the number of data packets sent. As QoS-GRACO aims to deliver data while respecting its QoS requirements including the end-to-end delay, data is delivered more quickly depending on the class of traffic. Indeed, packets belonging to $C_{0} T_{1}$, being the most constrained class of traffic, are transmitted faster than the packets from other less demanding classes $\left(\mathrm{CoT}_{2}, \mathrm{CoT}_{3}, \mathrm{CoT}_{4}\right)$. Similarly for the $\mathrm{CoT}_{2}$ class, the packets are transmitted faster the ones from $\mathrm{CoT}_{3}$ and $\mathrm{CoT}_{4}$, and so on.

When comparing the end-to-end delays per packet, presented on Fig. 6b, we realise that the first data packets sent of each class always take more time to be delivered, in fact, QoS-GRACO launches the recovery phase the first time a packet is sent to a destination when it arrives to a void, whether physical void or QoS void, however, the following data packets sent to the same destination will use the pheromone trails dropped on the paths found during the previous recoveries. The dashed lines in Fig. $6 \mathrm{~b}$

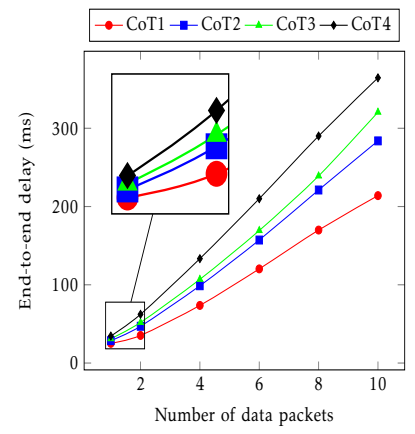

(a) End-to-end delay varying with the number of data packets sent

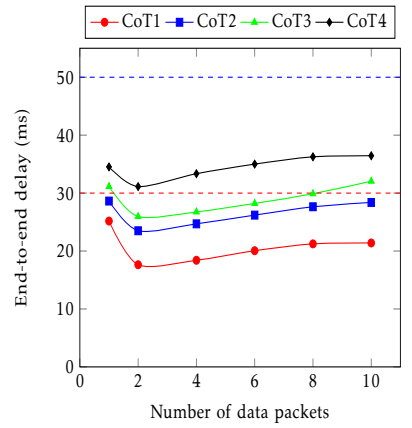

(b) End-to-end delay per packet varying with the number of data packets sent
Fig. 6: End-to-end delay - 802.15.4 MAC

represent the limits of the required time of $\operatorname{CoT}_{1}$ and $\mathrm{CoT}_{2}$ : the red line for $\operatorname{CoT}_{1}$, and the blue line for $\operatorname{CoT}_{2}$. The limits of the required time of $\mathrm{CoT}_{3}(200 \mathrm{~ms})$ and $\mathrm{CoT}_{4}$ (1s) are not presented on the figure because the values are out of the range of values of the figure. Note that the time of all classes are relatively small, this is due to the possibility of using paths with pheromones of alternatives colours, especially for less demanding classes like $\mathrm{CoT}_{4}$ class. Indeed, packets of this class can use all the colours of pheromones, so they can get to their destination in a shorter time compared to the required delay.

\section{Data delivery rate}

Fig. 7a shows the delivery rates of QoS-GRACO using ideal and realistic MAC layers of the packets delivered with satisfying QoS requirements from the successfully delivered packets. Using Ideal for MAC, QoS-GRACO is capable of transmitting all data packets successfully and 

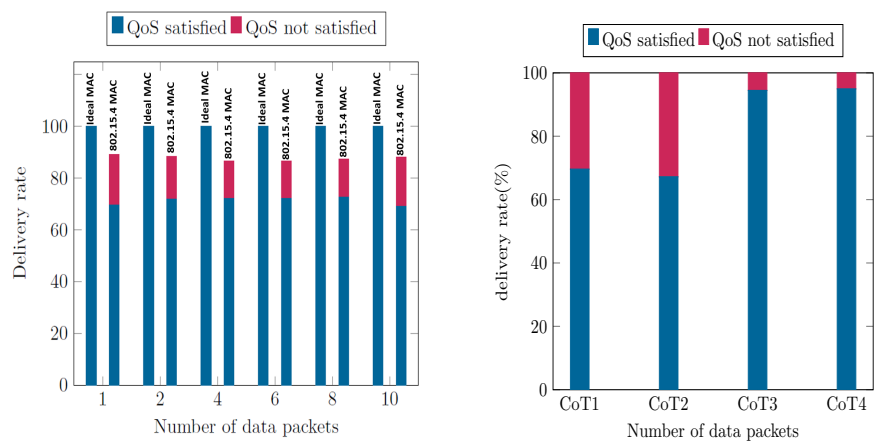

(a) Data delivery rate and packets (b) rate of data packet satisfying respecting QoS - Ideal MAC and the required QoSs - 802.15.4 MAC 802.15.4 MAC

Fig. 7: Data delivery rate

more, with the QoS required. With IEEE 802.15.4 MAC, the data delivery rate is maintained close to $90 \%$. Also the number of packets delivered respecting QoSs is affected, $70 \%$ of the packets arrive at their destination within the required delay and reliability level. Indeed, using a realistic MAC layer, the effect of multiple collisions that may occur at the MAC layer impact the reliability of links and the delays of sending some packets.

Fig. $7 \mathrm{~b}$ illustrates the delivery rate of data packets satisfying the required QoS among the data packets successfully delivered depending of the class of traffic. The less constrained classes of traffic have higher delivery. This is due to the instability of links when using realistic MAC layer. In particular, with the multiple collisions that may occur at the MAC layer, it is difficult to maintain links with high reliability and very restricted delays.

\section{Conclusion}

In this paper, we proposed a classification of NAN traffic based on its communication requirements in terms of reliability and delay. Then, based on this classification, we proposed QoS-GRACO, a routing protocol derived from GRACO [15], that takes account of the QoS requirements of NAN applications. QoS-GRACO is a geographical routing protocol combining a greedy phase and a recovery phase. A data packet is first routed using a modified greedy routing that accounts for the pheromones trails and the required QoS. During this mode, a node will progress the data packet toward its destination using geographic positions and QoS. In the case where a node can not find a path that meets the requirements of a packet using the first mode, the algorithm triggers a recovery phase. This second phase is also inspired from the recovery mechanism based on the ACO and the concept of zone proposed in GRACO. In order to take QoS requirements into account, we introduced the concept of coloured pheromone, the recovery process makes it possible to look for routes and mark them according to their quality. The recovery phase is also launched when the greedy is not possible when facing a physical void.

QoS-GRACO being a geographical routing protocol, it is local and distributed, it is then able to adapt to the network topology changes. Moreover, thanks to the efficiency of the recovery process, the protocol provides self-healing capability, which enhances its flexibility and scalability. Simulation results showed that QoS-GRACO is able to meet NAN QoS requirements in terms of end-oend delay and reliability. For the continuity of this work, we will improve the QoS model added to the protocol, by taking into account other QoS metrics. Also, it will be interesting to study other possible improvements in order to maximize the protocol efficiency by increasing the global data delivery rate and the delivery rate of packets satisfying the QoS requirements.

\section{REFERENCES}

[1] NIST OpenSG SG-Network Task Force Core Development Team. Smart grid networks system requirements specification release version 5 final. 2012.

[2] A. Zaballos, D. Vernet, and J. M. Selga. A genetic QoS-aware routing protocol for the smart electricity networks. Int. Journal of Distributed Sensor Networks, 2013.

[3] J. Ben-Othman, L. Mokdad, and M. O. Cheikh. Q-HWMP: improving end-to-end QoS for $802.11 \mathrm{~s}$ based mesh networks. In IEEE Global Telecommunications Conf., 2010.

[4] R. G. Garroppo, S. Giordano, and L. Tavanti. Implementation frameworks for IEEE 802.11 s systems. Computer Comm., 2010.

[5] X. Deng, L. He, X. Li, Q. Liu, L. Cai, and Z. Chen. A reliable QoS-aware routing scheme for neighbor area network in smart grid. Peer-to-Peer Networking and Applications, 2016.

[6] J. S. Jung, K. W. Lim, J. B. Kim, Y. B. Ko, Y. Kim, and S. Y. Lee. Improving IEEE 802.11 s wireless mesh networks for reliable routing in the smart grid infrastructure. In IEEE Int. Conf. on Comm. Workshops, 2011.

[7] J. Kim, D. Kim, K. W. Lim, Y. B. Ko, and S. Y. Lee. Improving the reliability of IEEE 802.11 s based wireless mesh networks for smart grid systems. Journal of Comm. and Networks, 2012.

[8] X. Deng, L. He, C. Zhu, M. Dong, K. Ota, and L. Cai. QoS-aware and load-balance routing for IEEE 802.11s based neighborhood area network in smart grid. Wireless Personal Comm., 2016.

[9] Y. Rajalingham, G.and Gao and T. Ho, Q.D.and Le-Ngoc. Quality of service differentiation for smart grid neighbor area networks through multiple RPL instances. In Proc. of the ACM symposium on QoS and security for wireless and mobile networks, 2014.

[10] V. C. Gungor, B. Lu, and G. P. Hancke. Opportunities and challenges of wireless sensor networks in smart grid. IEEE Transactions on Industrial Electronics.

[11] J. Gao, Y. Xiao, J. Liu, W. Liang, and CL P. Chen. A survey of communication/networking in smart grids. Future Generation Computer Systems, 2012.

[12] B. Karp and H. T. Kung. Gpsr: Greedy perimeter stateless routing for wireless networks. In Conf. on Mobile Computing and Networking, 2000.

[13] Q. Ho, G. Rajalingham, and T. Le-Ngoc. Performance and applicability of geographic-based routing in smart grid's neighbor area networks. In IEEE Int. Conf. on Advanced Technologies for Comm., 2013.

[14] M. Rekik, Z. Chtourou, and N. Mitton. Geographic routing protocol for peer-to-peer smart grid neighborhood area network. In IEEE Int. Conf. on Environment and Electrical Engineering, 2015.

[15] M. Rekik, N. Mitton, and Z. Chtourou. Geographic GReedy routing with ACO recovery strategy GRACO. In Int. Conf. on Ad Hoc Networks and Wireless. Springer, 2015.

[16] M. Thottan K.C. Budka, J.G. Deshpande. Communication Networks for Smart Grids Making Smart Grid Real. Springer, 2014.

[17] T. Sato, D. M. Kammen, B. Duan, M. Macuha, Z. Zhou, J. Wu, M. Tariq, and S. A. Asfaw. Smart Grid Standards: Specifications, Requirements, and Technologies. John Wiley \& Sons, 2015.

[18] US DOE. Communications requirements of smart grid technologies. US Department of Energy, Tech. Rep, 2010.

[19] G. G Finn. Routing and addressing problems in large metropolitan-scale internetworks. isi research report. 1987. 